\title{
EXACT AND UNIQUE SOLUTION OF A TRANSPORT EQUATION IN A SEMI-INFINITE MEDIUM BY LAPLACE TRANSFORM AND WIENER-HOPF TECHNIQUE
}

\author{
Z. ISLAM, A. MUKHERJEE AND S. KARANJAI
}

\begin{abstract}
The equation of radiative transfer in non-conservative case for diffuse reflection in a plane-parallel semi-infinite atmosphere with axial symmetry has been solved by Laplace transform and Wiener-Hopf technique. We have determined the emergent intensity in terms of Chandrasekhar's H-function and the intensity at any optical depth by inversion.
\end{abstract}

\section{Basic Equation and Solution for Emergent Intensity}

A parallel beam of radiation of net flux HF per unit area nomral to itself is incident on a plane parallel atmosphere of semi-infinite thickness in some specified direction $\left(-\mu, \phi_{0}\right)$. The equation appropriate to the problem (Chandrasekhar [2]; DasGupta [3]) is

$$
\mu \frac{d I(t, \mu)}{d t}=I(t, \mu)-\frac{1}{2} \int_{-1}^{1} p\left(\mu, \mu^{\prime}\right) I\left(t, \mu^{\prime}\right) d \mu^{\prime}-\frac{F}{4} e^{-\frac{t}{\mu_{0}}} p\left(\mu,-\mu_{0}\right)
$$

where $I(t, \mu)$ is the intensity characterizing the diffuse radiation field in the direction $\cos ^{-1} \mu$ at the optical depth $t$. We take (Busbridge [1], art.45; Chandrasekhar [2])

$$
p\left(\mu, \mu^{\prime}\right)=\omega_{0}+\omega_{1} \mu \mu^{\prime} .
$$

The average intensity $J_{0}(t)$ and the normal flux $J_{1}(t)$ are defined by

$$
J_{r}(t)=\frac{1}{2} \int_{-1}^{1} \mu^{r} I(t, \mu) d \mu ; \quad r=0,1 .
$$

Boundary conditions are

$$
I(0,-\mu)=0, \quad 0<\mu \leq 1
$$

and

$$
I(t, \mu) e^{-\frac{t}{\mu}} \rightarrow \infty \text { as } t \rightarrow \infty, \quad|\mu|<1 .
$$

Received June 5, 2003; revised February 16, 2004

2000 Mathematics Subject Classification. 85A25.

Key words and phrases. Radiative transfer, Wiener-Hopf technique. 
Equation (1) takes the form

$$
\mu \frac{d I(t, \mu)}{d t}=I(t, \mu)-\omega_{0} J_{0}(t)-\omega_{1} \mu J_{1}(t)-\frac{F}{4}\left(\omega_{0}-\omega_{1} \mu \mu_{0}\right) e^{-\frac{t}{\mu_{0}}} .
$$

Laplace transform of equation (6) gives

$$
(\mu s-1) I^{*}(s, \mu)=\mu s I(0, \mu)-\omega_{0} J_{0}^{*}(s)-\omega_{1} \mu J_{1}^{*}(s)-\frac{F}{4}\left(\omega_{0}-\omega_{1} \mu \mu_{0}\right) \frac{\mu_{0} s}{1+\mu_{0} s} .
$$

The formal solution of equation (6) gives, setting $\mu=\frac{1}{s}$, is

$$
I\left(0, \frac{1}{s}\right)=\omega_{0} J_{0}^{*}(s)+\frac{\omega_{1}}{s} J_{1}^{*}(s)+\frac{F}{4}\left(\omega_{0}-\frac{\omega_{1} \mu_{0}}{s}\right) \cdot \frac{\mu_{0} s}{1+\mu_{0} s}
$$

Multiplying equation (8) by $\frac{1}{2} d \mu$ and $\frac{1}{2} \frac{d \mu}{\mu s-1}$ successively and integrating between -1 and 1 and eliminating $J_{0}^{*}(s), J_{1}^{*}(s)$

$$
T(z) I(0, z)=G^{+}(z)+\frac{F \mu_{0}}{4\left(\mu_{0}+z\right)}\left[\omega_{0}-\left(\omega_{1} \mu_{0}-\omega_{0} \omega_{1} \mu_{0}\right) z\right]
$$

where

$$
\begin{aligned}
T(z)= & 1-2 z^{2} \int_{0}^{1} \frac{U(x)}{z^{2}-x^{2}} d x \\
U(x)= & \frac{1}{2}\left[\omega_{0}+\omega_{1}\left(1-\omega_{0}\right) x^{2}\right] \\
& \int_{0}^{1} U(x) d x<\frac{1}{2}
\end{aligned}
$$

and

$$
G^{+}(z)=\frac{1}{2} \int_{0}^{1} \frac{x}{x-z}\left[\omega_{0}+\omega_{1}\left(1-\omega_{0}\right) x z\right] I(0, x) d x .
$$

We now proceed to solve the integral equation (9).

Following Busbride [1] we have

$$
\frac{I(0, z)}{H(z)}\left(z+\mu_{0}\right) \frac{k-z}{k}=c_{0}+c_{1} z+c_{2} z^{2}
$$

where

$$
H(z)=1+z H(z) \int_{0}^{1} \frac{U(x) H(x)}{x+z} d x .
$$

Equation (14) gives the emergent intensity as

$$
I(0, z)=\frac{k\left(c_{0}+c_{1} z+c_{2} z^{2}\right)}{(k-z)\left(z+\mu_{0}\right)} H(z) .
$$




\section{Intensity at any Optical Depth}

The radiation intensity at any optical depth $t$ is given by

$$
I(t, \mu)=\frac{1}{2 \pi i} \lim _{\delta \rightarrow \infty} \int_{c-i \delta}^{c+i \delta} \frac{I^{*}(s, \mu)}{s} e^{s t} d s, \quad c>0 .
$$

The integrand of equation (17) has simple poles at $s=-\frac{1}{\mu_{0}}$ and $s= \pm k ; s=0$ is not a pole. Again

$$
\lim _{s \rightarrow \frac{1}{\mu}}\left(s-\frac{1}{\mu}\right) \frac{I^{*}(s, \mu)}{s} e^{s t}=0
$$

Therefore, $s=\frac{1}{\mu} \mathrm{f}$ is not a pole of the integrand of equation (17). The pole $s=-\frac{1}{\mu_{0}}$ is on the singular line and the residue must be calculated there. Hence the integrand of equation (17) is regular for $(-\infty,-1)^{c}$. Therefore, by Cauchy's residue theorem, equation (17) gives

$$
I(t, \mu)=R_{1}+R_{2}+R_{3}+\frac{1}{2 \pi i} \int_{H E} \frac{I^{*}(s, \mu)}{s} e^{s t} d s+\frac{1}{2 \pi i} \int_{D A} \frac{I^{*}(s, \mu)}{s} e^{s t} d s
$$

where

$$
\begin{aligned}
& R_{1}=-2 \frac{k e^{-\frac{t}{\mu_{0}}}}{\mu+\mu_{0}} \cdot \frac{\omega_{0}-\omega_{1}\left(1-\omega_{0}\right) \mu \mu_{0}}{\omega_{0}+\omega_{1}\left(1-\omega_{0}\right) \mu_{0}^{2}} \cdot \frac{c_{0}-c_{1} \mu_{0}+c_{2} \mu_{0}^{2}}{H\left(\mu_{0}\right)\left(k+\mu_{0}\right)} \cdot \frac{X\left(-\mu_{0}\right)}{Z\left(-\mu_{0}\right)} \\
& R_{2}=\frac{\omega_{0}+\omega_{1}\left(1-\omega_{0}\right) k \mu}{\omega_{0}+\omega_{1}\left(1-\omega_{0}\right) k^{2}} \cdot \frac{c_{0}+c_{1} k+c_{2} k^{2}}{\left(k+\mu_{0}\right)(\mu-k)} \cdot k H(k) e^{\frac{t}{k}} \\
& R_{3}=\frac{k^{2}}{2} e^{-\frac{t}{k}} \frac{\omega_{0}-\omega_{1}\left(1-\omega_{0}\right) k \mu}{\omega_{0}+\omega_{1}\left(1-\omega_{0}\right) k^{2}} \cdot \frac{c_{0}-c_{1} k+c_{2} k^{2}}{(k+\mu)\left(\mu_{0}-k\right)} \cdot \frac{1}{H(k)\left[\frac{d}{d s} T\left(\frac{1}{s}\right)\right]_{s=\frac{1}{k}}}
\end{aligned}
$$

where

$$
\begin{aligned}
\frac{d}{d s}\left[T\left(\frac{1}{s}\right)\right]_{s=\frac{1}{k}}= & \frac{k^{3}}{k^{2}-1}\left[\left(3 k^{2}-1\right)\left(1-\omega_{0}\right) \omega_{1}+\omega_{0}\right] \\
& +\frac{k^{2}}{2}\left[\omega_{0}+3 \omega_{1}\left(1-\omega_{0}\right) k^{2} \ln \frac{k-1}{k+1}\right]
\end{aligned}
$$

\section{Determination of the Constants $c_{0}, c_{1}, c_{2}$}

We rewrite the equation (9) in the form

$$
\left(\frac{k-z}{k}\right) T(z) I(0, z)\left(\mu_{0}+z\right)=\frac{k-z}{k}\left(\mu_{0}+z\right) G^{+}(z)+\frac{F \mu_{0}}{4}\left[\omega_{0}-\omega_{1}\left(1-\omega_{0}\right) \mu_{0} z\right] \frac{k-z}{k} .
$$

Again $\frac{1}{H(-z)} \rightarrow\left(1-h_{0}\right)-\frac{h_{1}}{z}-\frac{h_{2}}{z^{2}}-\cdots$ as $z \rightarrow \infty$ 
where

$$
h_{r}=\int_{0}^{1} x^{r} U(x) H(x) d x ; \quad r=0,1,2, \ldots
$$

We substitute the expression for $I(0, z)$ from equation (16) in equation (24) and equating the coefficients of $z^{0}, z$ and $z^{2}$ from bothsides we obtain

$$
\begin{aligned}
& {\left[\left(1-h_{0}\right)+\frac{k \mu_{0}}{2} \omega_{1}\left(1-\omega_{0}\right) \alpha_{1}+\left(k-\mu_{0}\right) d_{1}-d_{2}\right] c_{0}} \\
& +\left[-h_{1}+\frac{k \mu_{0}}{2} \omega_{1}\left(1-\omega_{0}\right) \alpha_{2}+\left(k-\mu_{0}\right) d_{2}-d_{3}\right] c_{1} \\
& +\left[-h_{2}+\frac{k \mu_{0}}{2} \omega_{1}\left(1-\omega_{0}\right) \alpha_{3}+\left(k-\mu_{0}\right) d_{3}-d_{4}\right] c_{2}=\frac{1}{4} F \omega_{0} \mu_{0} \\
& {\left[\frac{1}{2}\left(k-\mu_{0}\right) \omega_{1}\left(1-\omega_{0}\right) \alpha_{1}-d_{1}\right] c_{0}+\left[-\left(1-h_{0}\right)+\frac{1}{2}\left(k-\mu_{0}\right) \omega_{1}\left(1-\omega_{0}\right) \alpha_{2}+d_{2}\right] c_{1}} \\
& +\left[h_{1}+\frac{1}{2}\left(k-\mu_{0}\right) \omega_{1}\left(1-\omega_{0}\right) \alpha_{3}+d_{3}\right] c_{2}=\frac{1}{4} F \omega_{0}\left[\frac{\omega_{0}}{k}+\omega_{1}\left(1-\omega_{0}\right) \mu_{0}\right] \\
& \frac{1}{2} \omega_{1}\left(1-\omega_{0}\right) \alpha_{1} c_{0}+\frac{1}{2} \omega_{1}\left(1-\omega_{0}\right) \alpha_{2} c_{1}+\left[\frac{1}{2} \omega_{1}\left(1-\omega_{0}\right) \alpha_{3}-\left(1-h_{0}\right)\right] c_{2} \\
& =\frac{1}{4} F \frac{\mu_{0}^{2}}{k} \omega_{1}\left(1-\omega_{0}\right)
\end{aligned}
$$

where

$$
\alpha_{r}=\int_{0}^{1} \frac{x^{r} H(x)}{(k-x)\left(\mu_{0}+x\right)} d x ; \quad r=1,2,3 .
$$

and

$$
d_{r}=\int_{0}^{1} \frac{x^{r} U(x) H(x)}{(k-x)\left(\mu_{0}+x\right)} d x ; \quad r=1,2,3,4 .
$$

Equations (26), (27) and (28) determine the constants $c_{0}, c_{1}, c_{2}$ explicitly.

\section{References}

[1] I. W.Busbridge, The Mathematics of Radiative Transfer, Cambridge University Press, Cambridge, 1960.

[2] S. Chandrasekhar, Radiative Transfer, Dover Publications, New York, 1960.

[3] S. R. DasGupta, Astrophysics Space Science, 50, 187, 1977.

Department of Mathematics, Malda College, Malda, West Bengal, 732101, India.

Department of Mathematics, North Bengal University, West Bengal, 734430, India. 